\title{
La incontinencia urinaria
} Urinary incontinence

\author{
J. E. Robles
}

\section{RESUMEN}

La incontinencia urinaria, entendida como cualquier pérdida involuntaria de orina, constituye un problema médico y social importante. Puede clasificarse en incontinencia urinaria de esfuerzo, incontinencia urinaria de urgencia e incontinencia urinaria mixta, principalmente. Las proporciones de estos tres tipos principales de incontinencia urinaria son difíciles de establecer y varían notablemente según las fuentes, pero, podrían estar en torno a 40,33 y $20 \%$, respectivamente.

Su diagnóstico requiere una correcta historia clínica y exploración física, junto con algunas exploraciones complementarias.

El primer escalón terapéutico lo constituyen las medidas higiénico-dietéticas y las técnicas de modificación de la conducta. El tratamiento farmacológico es específico para cada tipo de incontinencia urinaria, utilizándose anticolinérgicos e inhibidores de la recaptación de serotonina.

Por último, las diferentes técnicas quirúrgicas tienen su papel ante el fracaso de los tratamientos conservadores o frente a incontinencia urinaria severa.

Palabras clave. Incontinencia urinaria. Diagnóstico. Tratamiento conservador. Tratamiento farmacológico. Cirugía.

\begin{abstract}
Urinary incontinence, understood as any involuntary loss of urine, constitutes an important medical and social problem. It can be classified as stress urinary incontinence, urgent urinary incontinence or mixed urinary incontinence. The proportions of these three types of urinary incontinence are difficult to establish and vary notably between sources, but they might be about 40,33 and $20 \%$ respectively.
\end{abstract}

Its diagnosis requires a correct clinical history and physical exploration, together with some complementary explorations.

The first therapeutic step consists of hygienicdietary measures and behaviour modification techniques. Pharmacological treatment is specific for each type of urinary incontinence, using anticholinergics and inhibitors of serotonin reuptake.

Finally, different surgical techniques have a role in cases where conservative treatments fail or when dealing with severe urinary incontinence.

Key words. Urinary incontinence. Diagnosis. Conservative treatment. Pharmacological treatment. Surgery.
Departamento de Urología. Clínica Universitaria de Navarra. Pamplona.

Aceptado para su publicación el 17 de febrero de 2006.

\author{
Correspondencia: \\ José Enrique Robles \\ Departamento de Urología \\ Clínica Universitaria de Navarra \\ Avda. Pío XII, 36 \\ 31008 Pamplona \\ E-mail: jerobles@unav.es
}




\section{INTRODUCCIÓN}

La incontinencia urinaria (IU) constituye un problema médico y social importante con una tendencia creciente debido, entre otras razones, al envejecimiento de la población. Recientes estimaciones cifran en 200 millones los adultos con IU.

Aunque existen diferentes definiciones de IU, la tendencia actualmente es tratar de homogeneizar éstas y seguir el concepto propuesto por la Sociedad Internacional de Continencia (ICS). De acuerdo con ésta, la IU se define como cualquier pérdida involuntaria de orina ${ }^{1}$. Esta definición abarca varios aspectos de la incontinencia, incluyendo síntomas (utilizados en estudios epidemiológicos y ensayos clínicos que típicamente utilizan cuestionarios basados en síntomas), signos físicos (los más relevantes para los clínicos), la observación urodinámica y la condición en su conjunto ${ }^{2}$.

Es altamente prevalente en la población adulta y de dos a cuatro veces más común en mujeres que en hombres. La incidencia de IU aumenta casi linealmente con la edad hasta ser considerada como uno de los síndromes geriátricos tanto por su elevada prevalencia en mayores de 65 años como por el impacto negativo que ocasiona en el anciano que la sufre ${ }^{3}$.

$\mathrm{Al}$ mismo tiempo es sorprendentemente bajo el índice de consulta generado por este problema de salud ${ }^{4}$ y la mayoría de los pacientes que viven en la comunidad utilizan productos absorbentes, influyendo en ello factores individuales, socioculturales y puramente asistenciales ${ }^{5}$.

La IU no es obviamente un proceso de riesgo vital, pero deteriora significativamente la calidad de vida de los pacientes, limita su autonomía y reduce su autoesti$\mathrm{ma}^{6}$. La afectación de la calidad de vida relacionada con la salud (CVRS) por la IU puede incluso ser mayor que la provocada por algunas enfermedades crónicas como la diabetes o la hipertensión arterial.

Las cifras de prevalencia que aparecen en los diferentes estudios son muy variables, dependiendo de una serie de factores como concepto de incontinencia, método de estudio, nivel asistencial estudiado y otras características de la muestra ${ }^{4}$.
En estudios epidemiológicos entre mujeres adultas de todas las edades las tasas de prevalencia de IU oscilan entre 20 y $50 \%$.

En el estudio epidemiológico EPINCONT realizado en 27.936 mujeres mayores de 20 años en un condado de Noruega entre 1995 y $1997,25 \%$ de ellas confesaban tener pérdidas de orina ${ }^{7}$.

Un estudio reciente en cuatro países europeos (Francia, Alemania, Reino Unido y España) llevado a cabo sobre las respuestas de una encuesta por correo a 17.080 mujeres de más de 18 años demostró que 35\% de ellas referían pérdidas involuntarias de orina en los 30 días previos. La tasa de prevalencia fue muy similar en todos los países (41 a 44\%), excepto en España cuya tasa resultó sorprendentemente menor (23\%).

En ambos estudios se constata claramente que la prevalencia de IU aumenta con la edad, con una tasa típica en adultos jóvenes de $20-30 \%$, un pico alrededor de la edad media (prevalencia 30-40\%) y luego un incremento leve y mantenido en edades más avanzadas (prevalencia 30-50\%) ${ }^{5,7,8}$.

Los pacientes institucionalizados, en particular las mujeres, representan un grupo particular de elevada prevalencia de $\mathrm{IU}$, alrededor de $50-60 \%^{3,9}$.

\section{TIPOS DE INCONTINENCIA URINARIA}

Se considera que la IU y las disfunciones miccionales pueden clasificarse de acuerdo con criterios sintomáticos o urodinámicos, según las directrices de la ICS en un documento de estandarización de la terminología ${ }^{1}$. Según los criterios sintomáticos, los tipos fundamentales de IU se exponen a continuacción:

\section{Incontinencia urinaria de esfuerzo}

La IU de esfuerzo (IUE) se define como la pérdida involuntaria de orina asociada a un esfuerzo físico que provoca un aumento de la presión abdominal (como por ejemplo toser, reír, correr, andar). Se produce cuando la presión intravesical supera la presión uretral como consecuencia de un fallo en los mecanismos de resistencia uretral, por dos causas no excluyentes: 
- Por hipermovilidad uretral, en el que fallan los mecanismos de sujeción de la uretra que desciende de su correcta posición anatómica.

- Por deficiencia esfinteriana intrínseca, en la que existe una insuficiente coaptación de las paredes uretrales que produce una disminución de la resistencia de la uretra.

La IUE es común en ancianas y supone también la forma más común de IU en las mujeres menores de 75 años, afectando a casi un $50 \%$ de ellas ${ }^{5,10}$.

En el varón anciano es rara y por lo común está asociada a cirugía prostática previa, tanto transuretral como suprapúbica ${ }^{4}$.

\section{Incontinencia urinaria de urgencia}

La IU de urgencia (IUU) es la pérdida involuntaria de orina acompañada o inmediatamente precedida de "urgencia". Por "urgencia" se entiende cuando el paciente se queja de la aparición súbita de un deseo miccional claro e intenso, difícil de demo$\operatorname{rar}^{11}$. Esta sensación de urgencia es consecuencia de una contracción involuntaria del detrusor vesical. Cuando estas contracciones involuntarias se ponen de manifiesto en un estudio urodinámico se denomina "detrusor hiperactivo", cuya causa puede ser una enfermedad neurológica (hiperactividad del detrusor neurogénica) o sin causa detectable (hiperactividad del detrusor idiopática). Puede además deberse a otras causas, como estenosis uretral, hipercorrección quirúrgica, etc.

Es la forma más común de IU en mayores de 75 años, sobre todo en los hombres $(40-80 \%)^{4,8}$.

En los grandes estudios epidemiológi$\cos \operatorname{citados}^{6,7}$ entre 11 y $20 \%$ de las mujeres incontinentes tenían síntomas de IUU.

\section{Incontinencia urinaria mixta}

$\mathrm{La}$ incontinencia urinaria mixta (IUM) es la percepción de pérdida involuntaria de orina asociada tanto a urgencia como al esfuerzo. Se estiman en $30-40 \%$ las mujeres con IU que tienen síntomas de IUM ${ }^{7}$. Estos datos confirman previos estudios en los que se concluía que la IUE es un problema frecuente en mujeres jóvenes y premenopáusicas, mientras que los síntomas de urgencia y la IUU se hacen más predominantes en el rango de edad postmenopáusica ${ }^{4}$.

\section{Incontinencia urinaria continua}

IU continua (IUC) es la pérdida involuntaria y continua de orina. Puede ser debida a una fístula, desembocadura ectópica ureteral o a un déficit intrínseco uretral grave.

\section{Enuresis nocturna}

Enuresis nocturna es la pérdida involuntaria de orina durante el sueño.

\section{Incontinencia urinaria inconsciente}

La incontinencia urinaria inconsciente (IUI) se define como la pérdida involuntaria de orina sin deseo miccional e independientemente de cualquier aumento de la presión abdominal. Este tipo de IU se presenta sólo con grandes volúmenes de orina dentro de la vejiga y aunque poco frecuente es de interés reconocerla entre la población geriátrica. Desde el punto de vista etiológico es una forma extrema de disfunción de vaciado vesical, bien por una obstrucción en el tracto urinario inferior (crecimiento prostático, esclerosis del cuello vesical) o por un detrusor acontráctil (neurológico o por otros factores) ${ }^{3}$.

\section{Otras incontinencias urinarias}

Otras formas de IU se pueden presentar de forma ocasional, como la IU durante el coito o la IU con la risa ("giggle incontinence"). Inmediatamente después de la micción la IU se describe con el término goteo postmiccional, que es la pérdida de orina que se percibe justo tras finalizar la micción, generalmente después de salir del baño.

\section{PREVALENCIA DE INCONTINENCIA URINARIA POR SUBTIPOS}

En los estudios epidemiológicos amplios anteriormente citados se muestra que la IUE es el síntoma de IU más frecuentemente referido. La IUE pura afecta al $10-20 \%$ de las mujeres incontinentes, mientras que $30-40 \%$ tienen síntomas de 
IUM $^{12}$. Como la IUM es la combinación de IUE y IUU, la mayoría de las mujeres, independientemente de su edad, se quejan de IUE con o sin otros síntomas del tracto urinario inferior. Esto se corresponde con los porcentajes comunicados en el extenso estudio EPINCONT ${ }^{7}$, en el que $50 \%$ de las mujeres incontinentes tenían síntomas de IUE, $11 \%$ de IUU y $36 \%$ de IUM. Estos datos han sido confirmados en recientes revisiones ${ }^{10}$.

El porcentaje de mujeres incontinentes con IUE alcanza un pico máximo alrededor de la quinta década (rango, 28\% a $65 \%)^{5,10}$ y luego declina a partir de la sexta década. Por contraste, la IUM es más frecuente en mujeres mayores y supone un 40 a $48 \%$ en el rango de 60 años en adelante ${ }^{5}$.

Es importante tener en cuenta que estos estudios epidemiológicos están basados en los síntomas que la paciente registra en la encuesta y es más que probable que esto sobredimensione la IUM y la IUU en detrimento de la IUE4 .

\section{SEVERIDAD DE LA INCONTINENCIA URINARIA}

En muchos estudios sobre IU la definición de ésta es elusiva acerca de la severidad, porque no se tienen en cuenta ni la frecuencia de las pérdidas ni su cantidad. En tal sentido se ha desarrollado un "índice de severidad" validado y que ha puesto de manifiesto que en sólo $27 \%$ de las mujeres incontinentes esta IU era severa, mientras que más del $50 \%$ tenían IU leve ${ }^{13}$.

La severidad de la IU se incrementa con la edad ${ }^{9}$. En el estudio EPINCONT se demuestra que la IU severa supone el 17, 28 y $38 \%$ de la IUE, IUU y IUM, respectivamente. En lo referente al grupo de IUE la severidad de la incontinencia aumentaba de $10 \%$ en el grupo de edad 25 a 44 años a $33 \%$ en el de 60 ó más años. Para la IUU y la IUM los valores correspondientes fueron 8 a 45 y 19 a $53 \%{ }^{6}$. El citado estudio epidemiológico definió la IU significativa como aquella de los pacientes con moderada o severa IU por el índice de severidad. La prevalencia de esta IU significativa aumenta con la edad, con independencia del tipo de IU, con un rango de $1,7 \%$ en la tercera década de la vida a $16,2 \%$ en el grupo de pacientes mayores de 85 años ${ }^{6}$.

\section{FACTORES DE RIESGO DE LA INCONTINENCIA URINARIA}

Existen en la literatura varios estudios acerca de los putativos factores de riesgo que pudieran incrementar el riesgo individual de desarrollar IU.

\section{Género}

Varias razones (longitud uretral, diferencias anatómicas en el suelo pelviano, efectos del parto) intentan justificar la diferencia de que las mujeres tengan un mayor riesgo de desarrollar IU. Sin embargo, esta razón de riesgo varía con la edad: en menores de 60 años las mujeres tienen cuatro veces más probabilidades que los hombres de padecer IU, pero en mayores de 60 años sólo dos veces más ${ }^{9}$.

\section{Edad}

Existen indicios para pensar que la edad pudiera jugar un papel en el desarrollo de la IUU más que de la IUE. Los cambios relacionados con la edad que pudieran contribuir al desarrollo de IU se muestran en la tabla 1.

Tabla 1. Edad en incontinencia urinaria. Factores relacionados.
1. Disminución del tejido elástico
2. Atrofia celular
3. Degeneración nerviosa
4. Reducción tono músculo liso
5. Hipoestrogenismo
6. Deterioro cognitivo
7. Inmovilidad
8. Pérdida de capacidad concentración renal
9. Comorbilidad: polifarmacia

\section{Menopausia y hipoestrogenismo}

La atrofia genital puede contribuir a la relajación del suelo pelviano así como a la deficiencia intrínseca del esfínter uretral. Estudios recientes, no obstante, no han demostrado que la menopausia por sí misma sea un factor de riesgo independiente de la edad ${ }^{13}$. 


\section{Raza}

Estudios multivariados demuestran que las mujeres blancas tienen una prevalencia de IUE tres veces mayor que las mujeres negras ${ }^{14}$, atribuyéndose a diferencias raciales en el tejido conjuntivo o en los músculos.

\section{Peso}

La obesidad es uno de los factores de riesgo independiente para la IU en mujeres mayores. Un índice de masa corporal (IMC) alto se correlaciona con una mayor prevalencia de IUE y de IUM, así como con la severidad de la IU. Se conoce poco sobre la pérdida de peso como tratamiento de la $\mathrm{IU}^{15}$.

\section{Paridad}

El parto es un factor establecido de riesgo de IUE y IUM entre las mujeres jóvenes y de mediana edad ${ }^{16}$. Se ha sugerido que el parto vaginal es el factor contribuyente más importante, posiblemente por las lesiones neurológicas o musculares asociadas. Sin embargo, el embarazo en sí mismo puede causar cambios mecánicos u hormonales que sean causa de IU ${ }^{9}(31-42 \%$ de las embarazadas tienen IUE y en $51 \%$ de ellas persiste algún grado de IU postparto). Por otro lado, recientemente ${ }^{17}$ se ha comprobado que las mujeres con partos por cesárea (prevalencia 15,8\%) tienen un riesgo mayor de IU que las nulíparas (prevalencia $10,1 \%$ ), pero que el parto vaginal se asocia con el riesgo mayor ( prevalencia $24,2 \%)$.

Se han estudiado varios parámetros obstétricos que pueden contribuir a la aparición de IU. En un reciente estudio ${ }^{18}$, los RN de peso 4.000 g o más se asociaron a cualquier tipo de IU e IUE; trastornos funcionales del parto con IU moderada o severa; la anestesia epidural con IUE y la circunferencia cefálica mayor de $38 \mathrm{~cm}$ con IUU. El resto de los parámetros estudiados (edad gestacional, instrumentación del parto, etc) no mostraron asociación significativa.

\section{Histerectomía}

El impacto de la histerectomía en el desarrollo de la IUE no está demostrado. Un ya clásico metaanálisis ${ }^{19}$ publicaba una asociación entre histerectomía e IU a largo plazo, es decir, muchos años tras la cirugía sobre todo en mujeres mayores de 60 años en las que el riesgo de IU se incrementaba hasta $60 \%$. Otros estudios, no encuentran esta asociación ${ }^{20}$.

\section{Factores uroginecológicos}

Los prolapsos de la pared vaginal y de los órganos pélvicos, la debilidad de los músculos del suelo pelviano, la cirugía uroginecológica previa, el estreñimiento, las cistitis y otras infecciones del tracto urinario son los factores uroginecológicos más importantes asociados a $\mathrm{IU}^{21}$.

\section{Otros factores}

Incluyendo cardiopatías, diabetes mellitus, enfermedad de Parkinson, demencia, accidentes cerebrovasculares, varios fármacos, tabaquismo, abuso del alcohol, etc, se ha relacionado con $\mathrm{IU}^{5}$.

Un estudio reciente ${ }^{22}$ ha encontrado una prevalencia de 71 y $24 \%$ de IUE entre madres y hermanas de mujeres operadas por IU, frente a 40 y $11 \%$ de madres y hermanas de mujeres no operadas, hallándose además un inicio de los síntomas en edad más joven entre las primeras. Además, datos extraídos del estudio EPINCONT ponen de manifiesto que las mujeres son proclives a desarrollar IU si sus madres o hermanas mayores son incontinentes ${ }^{23}$. Todo ello hace pensar que la herencia puede jugar algún papel en la IUE.

\section{IMPACTO DE LA IU EN LA CALIDAD DE VIDA}

Varios estudios han tratado de medir la calidad de vida relacionada con la salud (CVRS) en las mujeres incontinentes. Estos estudios varían en su diseño, metodología, criterios de diagnóstico de IU e incluso definición de calidad de vida. Sin embargo, en todos ellos se expone que la IU tiene un impacto negativo notable sobre múltiples aspectos de la vida diaria, tanto en el entorno social (menor interacción social o mayor aislamiento, abandono de algunas aficiones, etc), como en el físico (limitaciones a la práctica deportiva), sexual (pérdida de la actividad sexual, evitación de la pareja), psi- 
cológico (pérdida de la autoestima, apatía, depresión, sentimientos de culpabilidad, etc), laboral (absentismo, menos relación) y doméstico (precauciones especiales con la ropa, protección de la cama, etc) ${ }^{24}$.

Las mujeres con IU desarrollan hábitos de comportamiento para paliar el problema en parte, tales como la reducción en la ingesta de líquidos, el aislamiento y retracción social, la utilización de absorbentes y la adquisición de una determinada frecuencia miccional, y otros hábitos miccionales, voluntaria ${ }^{25}$.

La IU afecta más a la CVRS de las más jóvenes y está relacionada con el tipo de IU de que se trate ${ }^{26}$.

La ICS recomienda incluir parámetros de calidad de vida en la evaluación de los tratamientos de la IU utilizando cuestionarios mixtos de síntomas y CV. Existen dos cuestionarios específicos para evaluar la CV de mujeres con IU validados en España. Uno es el King's Health Questionnaire ${ }^{27}$ y el otro es la versión española del cuestionario de la International Consultation on Incontinence Short Form (ICIQ-SF) ${ }^{28}$, que es el primer cuestionario breve que permite no sólo detectar la IU sino además categorizar las pacientes de acuerdo con la gravedad de los síntomas y el grado de preocupación que éstos les generan. Así se termina poniendo de manifiesto que el impacto sobre CVRS depende del estilo de vida, la edad, el nivel sociocultural, y las experiencias personales no tanto por la gravedad de los síntomas.

\section{DIAGNÓSTICO CLÍNICO DE LA INCONTINENCIA URINARIA}

Los métodos básicos disponibles en todos los niveles asistenciales para el correcto diagnóstico de la IU son la anamnesis, la exploración física y el diario miccional.

Una detallada anamnesis, correctamente enfocada no sólo hacia el tipo de síntomas sino también hacia posibles factores de riesgo, permitirá intuir el tipo de incontinencia y, a partir de ahí, dirigir las pruebas diagnósticas complementarias en ese sentido.

En la tabla 2 se indican una serie de datos a valorar en la entrevista de una mujer que consulta por IU.
Tabla 2. Cuestionario de función urinaria. Frecuencia miccional.

- Nocturia

- Urgencia miccional

- Urgeincontinencia

- Emergencia

- IU espontánea

- IU con las relaciones sexuales

- IU inconsciente

- IU de esfuerzo

- Sensación de vaciamiento incompleto

- Disuria

- Dificultad miccional

- Dolor con la replección vesical

- Escozor miccional

- Infecciones urinarias

- Hematuria

- Estreñimiento

- Incontinencia fecal

- Síntomas de prolapso genital

Otro dato que debe recoger la historia clínica es el número de compresas o protectores utilizados al día por la paciente, como dato de valoración indirecta de la severidad de la IU.

Las características miccionales pueden evaluarse a partir de un diario miccional, en el que la paciente anota durante 3-7 días el intervalo y número de micciones al día, el volumen vaciado, los episodios de incontinencia y la causa (es decir, esfuerzo o urgencia $)^{29}$.

Es importante recoger también información acerca de la paridad, utilización de fórceps, episiotomías y duración del trabajo de parto, así como intervenciones previas para la corrección de la IU, intervenciones abdominales y pélvicas. Deben reflejarse así mismo otros factores de riesgo (sobrepeso, estreñimiento crónico, etc.) y fármacos y otras sustancias que pueden contribuir a la IU.

La exploración física debe hacerse con la vejiga llena, con la paciente en posición de litotomía dorsal. Incluye la evaluación de los prolapsos genitales asociados, una exploración neurológica básica de la zona lumbosacra para valorar el tono muscular del suelo 
pelviano y los reflejos bulbocavernoso y anocutáneo. La demostración del escape de orina debe hacerse primero en decúbito, haciendo toser a la paciente y observando el escape de orina por la uretra. Si no hay escapes debe repetirse en bipedestación.

El grado de hipermovilidad uretral se puede evaluar con el test del bastoncillo, introduciéndolo en la uretra y valorando el movimiento del mismo con la tos (positivo si es superior a $30^{\circ}$ ).

Finalmente, debe cuantificarse la orina residual mediante sondaje o ecografía.

Pocas pruebas de laboratorio son necesarias. Es imprescindible descartar infección urinaria mediante la realización de un cultivo de orina. No obstante, se consideran de utilidad como cribado las tiras reactivas, que tienen una elevada especificidad (92-100\%) pero variable sensibilidad (35$85 \%$ ) en el caso de la bacteriuria ${ }^{30}$.

\section{PRUEBAS DIAGNÓSTICAS COMPLEMENTARIAS}

\section{Estudio urodinámico}

Considerando que lo fundamental en el diagnóstico de IU en la mujer es identificar aquellas pacientes con IUU y distinguirlas de aquéllas que tienen IUE o IUM, y que el diagnóstico exacto de IUU sólo es posible con un método que permita objetivar la presencia de contracciones involuntarias del detrusor en la fase de llenado, se puede resumir que los estudios urodinámicos (Tabla 3) son imprescindibles en mujeres que consultan por $\mathrm{IU}^{31}$ :

- En el caso de una IUE pura (poco frecuente pues se estima en sólo 5-10\% IUE como único síntoma) la exploración urodinámica debe confirmar la IUE y excluir la hipoactividad del detrusor y la vejiga hiperactiva. Debe valorar la acomodación vesical y evaluar el patrón de vaciado y si está alterado, diferenciar una obstrucción vesical de una hipocontractilidad. Debe obtener una medida objetiva de la intensidad de la IUE mediante los puntos de presión de fuga o la presión máxima de cierre uretral, estableciendo, si lo hubiera, el diagnóstico de deficiencia esfinteriana intrínseca. Todo lo anterior es deter- minante en la elección del tratamiento apropiado.

- En pacientes con IUU es imprescindible una cistometría para evidenciar la presencia de un detrusor hiperactivo y sus características.

- En pacientes con IUM es fundamental para conocer el componente de IUE y de detrusor hiperactivo (evaluando la existencia o no de contracciones, la intensidad de las mismas, el volumen en el que se producen). Es importante también para dilucidar otras alteraciones funcionales menos claras (como obstrucción) enmascaradas detrás de supuestas IUM.

- Determinar la etiología de la IU en pacientes en las que ha fracasado el tratamiento conservador o farmacológico.

Tabla 3. Evaluación urodinámica de la incontinencia urinaria.

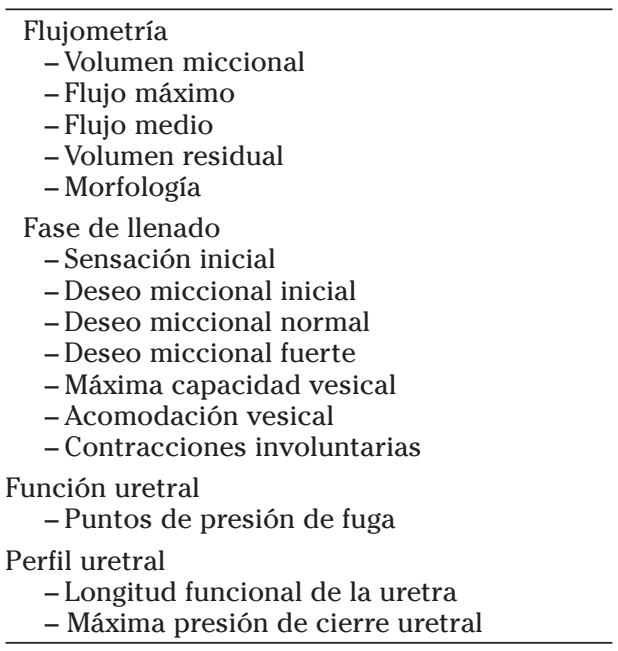

\section{Pruebas de imagen}

- Videocistouretrografía puede ser de utilidad en algunos pacientes, y combina medidas de la presión con imágenes radiológicas. Permite la evaluación de pacientes con disfunciones del tracto urinario inferior complicadas, generalmente debidas a alteraciones neurológicas ${ }^{29}$.

- Ecografía, aunque los estudios publicados utilizan infinidad de vías de acceso, distintos tipos de transductores y dis- 
tintas mediciones que hace difícil la validación de las técnicas y la comprobación de los resultados. No obstante, se han definido variables sencillas de medir (distancia uretra-cuello en reposo y esfuerzo) capaces de discernir entre continentes e incontinentes por hipermovilidad uretral con elevadas sensibilidad y especificidad ${ }^{32}$.

- Resonancia magnética, que en los últimos años ha ido adquiriendo gran importancia en el estudio anatómico y funcional del suelo pelviano ${ }^{33}$. No obstante, su indicación queda probablemente reducida a pacientes con IU y prolapso genital grande o complejo, en pacientes jóvenes y en aquellos con discrepancias entre los hallazgos clínicos y los métodos diagnósticos habituales ${ }^{22}$.

\section{TRATAMIENTO DE LA INCONTINENCIA URINARIA}

En el tratamiento de la IU se incluyen diversas alternativas, no excluyentes y a menudo complementarias. Para decidir el tratamiento más adecuado en cada paciente no sólo se deberá valorar el tipo de incontinencia sino también de las condiciones médicas asociadas, la repercusión de la IU, las preferencias de las pacientes y su tipo de vida, la aplicabilidad del tratamiento y el balance riesgo/beneficio de cada tratamiento.

\section{Tratamiento conservador de la incontinencia urinaria}

El tratamiento conservador de la IU agrupa diversos procedimientos y es considerado en la actualidad como un elemento esencial en el abordaje terapéutico. En no pocas ocasiones su aplicación es previa y/o simultánea a otras medidas de farmacológicas o quirúrgicas.

\section{Modificación del entorno}

Tratando de facilitar el acceso al cuarto de baño evitando las barreras físicas domésticas y disponer de sustitutivos (orinales, cuñas sanitarias, etc) o de algún tipo de adaptación. Esto es especialmente importante en pacientes con IUU o IUM fundamentalmente si tienen limitada su movilidad (ancianos).

\section{Medidas higiénico-dietéticas}

Un tratamiento aceptable en las mujeres con sobrepeso es la disminución del peso. En la actualidad se considera como una medida preventiva eficaz evitar la sobrecarga del suelo pelviano manteniendo un peso adecuado ${ }^{34}$.

Parece existir alguna evidencia que sugiere que las mujeres que realizan esfuerzos intensos con aumento de la presión presentan mayor riesgo de desarrollar IUE y prolapso genital ${ }^{35}$, por lo que en la actualidad se recomienda evitar este tipo de ejercicios.

Existe una asociación demostrada entre el hábito tabáquico y la $\mathrm{IU}^{34}$, quizá debido al aumento de presión abdominal en la fumadora crónica.

Una ingesta hídrica abundante, sobre todo en las horas previas al descanso nocturno, se relaciona con un mayor número de episodios de IU. Limitar esta ingesta a partir de la merienda y reducir el consumo de sustancias excitantes (alcohol, café, té) puede tener efectos beneficiosos sobre el número de escapes, aunque no hay datos concluyentes ${ }^{3,34}$.

Las medidas destinadas a disminuir el estreñimiento, el cambio en el tipo de prendas de ropa a utilizar (cómodo y con aperturas fáciles) o la modificación de aquellos fármacos que alteran la continencia (Tabla 4) pueden ser también medidas beneficiosas ${ }^{36}$.

Tabla 4. Fármacos que pueden provocar incontinencia urinaria.

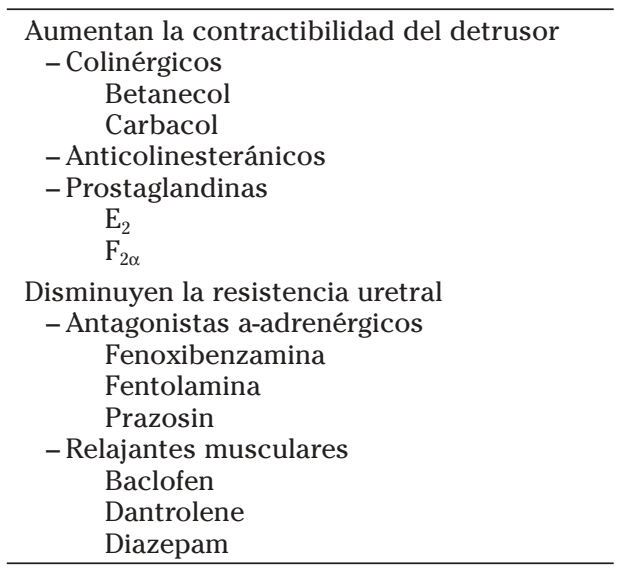




\section{Medidas paliativas}

En algunos pacientes se pueden utilizar los absorbentes como un elemento eficaz en el control de las consecuencias de la IU. Estos absorbentes permiten minimizar las repercusiones de la IU pero también pueden condicionar una falta de adecuado diagnóstico y tratamiento.

\section{Técnicas de modificación conductual}

Se agrupan bajo este nombre todas aquellas acciones destinadas a restablecer un patrón normal de vaciamiento vesical. Fundamentalmente, se basan en la realización de una micción programada, aumentando paulatinamente el intervalo entre micciones al instruir al paciente en disociar el deseo miccional de la acción de vaciado voluntario ${ }^{3}$. En este entrenamiento del vaciado vesical el diario miccional tiene un papel importante al permitir objetivar los progresos conseguidos.

Esta terapia conductual permite una disminución en la frecuencia de los escapes y en la severidad de la IU en alrededor de la mitad de los pacientes y en menor medida puede incluso recuperarse la continencia ${ }^{37}$.

\section{Rehabilitación muscular del suelo pelviano}

Se trata de un conjunto de técnicas dirigidas a mejorar el tono de la musculatura del suelo de la pelvis, favoreciendo un mejor soporte de las estructuras pélvicas y una adecuada movilidad uretral, favoreciendo asimismo la respuesta contráctil rápida y coordinada frente a los esfuerzos, que aumenta la presión de cierre uretral. Estos ejercicios musculares han revelado su eficacia en la IUE de la mujer tanto en la edad avanzada como en la mujer joven y puérpera, así como en la IUM ${ }^{38}$.

Este tipo de tratamiento conservador se considera indicado en mujeres con grados leve o moderado de IUE sin antecedentes de cirugía por IU, ni otro proceso concomitante ginecológico, neurológico o urológico ${ }^{6}$.

Existe una gran variedad de métodos para conseguir la mejoría y coordinación en la contracción de los músculos del suelo pelviano, incluyendo técnicas de biorretroalimentación ("bio-feedback") y electroestimulación ${ }^{39}$.
Los datos disponibles más recientes indican que la rehabilitación muscular del suelo pelviano reduce los episodios de IU en $54-72 \%$ y las tasas de curación/mejoría en ensayos aleatorizados varían entre 61 y $91 \%^{38}$. Con estos datos, es éste el tratamiento de primera opción en mujeres con IUE leve y moderada adecuadamente motivadas.

\section{Tratamiento farmacológico de la incontinencia urinaria}

En las fases de llenado y vaciado vesicales están involucradas una gran variedad de vías neuronales aferentes y eferentes (somáticas, simpáticas y parasimpáticas), reflejos y neurotransmisores centrales y periféricos (glutamato, serotonina, noradrenalina, dopamina, ácido gamma-amino-butírico, encefalina y acetilcolina). Las relaciones existentes entre todos estos factores no se conocen por completo, pero sí es bien conocido que la acetilcolina es el neurotransmisor periférico predominante y responsable de la contracción vesical ${ }^{35}$.

Los antimuscarínicos son los fármacos más empleados, entre otros, en el tratamiento de la IUU (Tabla 5). De estos fármacos se puede esperar que eliminen la incontinencia en el $20-30 \%$ de los casos y produzcan una mejora significativa de los síntomas en el $50 \%$ de los pacientes ${ }^{40}$. Los utilizados actualmente no muestran selectividad por los receptores muscarínicos vesicales, aunque su efecto parece más pronunciado en la vejiga que en otros órganos. Son estas reacciones las que limitan su utilización por los efectos secundarios, fundamentalmente sequedad de boca, ojos secos, estreñimiento, reflujo esofágico, visión borrosa, mareo y palpitaciones $^{40,41}$. Por ello, se están desarrollando nuevos agentes más selectivos para la vejiga, los últimos de los cuales son la darifenacina y la solifenacina, de reciente aparición en el mercado. Ambas han demostrado ser eficaces y bien toleradas, reduciendo significativamente la urgencia y los episodios de escape, con una baja incidencia de efectos secundarios, acompañándose de una baja tasa de abandonos de tratamiento, mejorando el cociente eficacia/tolerancia ${ }^{42}$. 
Tabla 5. Fármacos usados en el tratamiento de la incontinencia urinaria.

\begin{tabular}{l}
\hline Anticolinérgicos \\
- Oxibutinina \\
- Propiverina \\
- Tolterodina \\
- Trospio \\
Antagonistas a-adrenérgicos \\
- Alfuzosina \\
- Doxazosina \\
- Prazosin \\
- Tamsulosina \\
- Terazosina \\
Estrógenos \\
- Cremas vaginales \\
Otras \\
- Imipramina \\
- Desmopresina
\end{tabular}

Por otro lado, las monoaminas serotonina y noradrenalina están claramente implicadas en el mantenimiento del tono muscular y en los mecanismos de la continencia uretral. Los agonistas serotoninérgicos suprimen la actividad parasimpática y aumentan la actividad simpática y somática en el tracto urinario inferior, facilitando el llenado vesical. Basados en estas potenciales propiedades como promotoras de la continencia tanto de la serotonina como de la noradrenalina se han desarrollado varios estudios experimentales con inhibidores selectivos de la recaptación de ambos, a la cabeza de los cuales se sitúa la duloxetina ${ }^{43}$. Este fármaco es el primero de los aprobados para el tratamiento de la IUE en la mujer, y de los datos actuales se deduce que ocasiona un descenso en los episodios de incontinencia de 50$60 \%$, con un $56-74 \%$ de las mujeres tratadas que se sienten mejor tras el tratamiento ${ }^{44}$. El efecto adverso más significativo son las náuseas, aunque de intensidad leve a moderada, transitorias y no progresivas, que suelen desaparecer en un corto periodo de tiempo ${ }^{42}$. De momento está pendiente su aprobación en España con esta indicación.

\section{Tratamiento quirúrgico de la incontinencia urinaria}

La cirugía se considera el tratamiento adecuado en las pacientes en las que el tratamiento conservador ha fracasado o en aquellas con IUE moderada-severa. Su propósito es incrementar la resistencia uretral para evitar el escape de orina por la uretra durante los aumentos de la presión intraabdominal, preservando el vaciamiento vesical completo a baja presión.

Existen en torno a 200 procedimientos quirúrgicos diferentes para tratar la IUE, pero, pueden agruparse en tres tipos básicos: colposuspensiones, cabestrillos suburetrales e inyectables uretrales ${ }^{45}$.

\section{Inyectables uretrales}

Son el procedimiento menos invasivo y puede efectuarse con anestesia local. Inicialmente este procedimiento fue descrito para IUE por deficiencia esfinteriana intrínseca, aunque también puede ser eficaz en pacientes con hipermovilidad uretral. Una gran variedad de instancias se han utilizado, incluyendo colágeno, politetrafluroetileno, silicona, semillas de carbón, dextranómero y ácido hialurónico, e incluso tejidos autólogos como grasa, cada uno de ellos con diferentes propiedades biofísicas que afectan a la compatibilidad, tendencia a la migración, durabilidad y seguridad.

La mayor parte de ellos se inyectan de forma retrógrada vía transuretral o transvaginal, bajo control endoscópico, en el tejido periuretral y alrededor del cuello vesical.

En la actualidad, los datos disponibles sugieren, pero no prueban, su eficacia en cuanto a mejoría subjetiva y objetiva a corto plazo de la IUE ${ }^{46}$, con tasas variables de éxito (curación más mejoría) de 30-80\%, pero que disminuyen significativamente con el tiempo. En mujeres con importantes comorbilidades puede ser una opción útil teniendo en cuenta que serán necesarias 2 ó 3 inyecciones en 12 meses para obtener un resultado satisfactorio.

\section{Cabestrillos suburetrales}

Pueden subdividirse en cabestrillos "clásicos" y mallas "libres de tensión" tipo TVT.

En los "clásicos" se han utilizado tanto materiales autólogos (fascia lata, duramadre) como sintéticos (Mersilene, GoreTex). Su indicación esencial son aquellas pacientes con deficiencia esfinteriana intrínseca. Asimismo, están indicados en 
mujeres con cirugía previa para IU fracasada. Un lazo se pasa por completo bajo la uretra o el cuello vesical y se ancla luego anteriormente en algún lugar de la pared abdominal o de las estructuras pélvicas para estabilizar la uretra. Las tasas de curación (definida como continencia completa) se sitúan en $73-95 \%$ y las de éxito (mejoría o continencia) en el 64-100\% ${ }^{47}$. Los resultados parecen ser mejores en pacientes no tratadas previamente y las complicaciones más frecuentes son la disfunción de vaciado vesical con retención (1-4\% de las pacientes), hiperactividad vesical "de novo" (6-14\% de las pacientes) y erosión del cabestrillo (sobre todo los sintéticos) en vejiga, uretra y vagina ${ }^{45}$. En general, los materiales autólogos parecen estar asociados con una mayor tasa de éxito y con menor número de complicaciones.

La malla suburetral libre de tensión TVT se ha convertido en el procedimiento más popular en el tratamiento de la IU. Es menos agresivo que cualquiera de los otros procedimientos de cabestrillo, pudiendo incluso realizarse bajo anestesia local. Su propósito es restablecer la adecuada fijación de la uretra media al pubis, reforzando los ligamentos pubouretrales. Una cinta de polipropileno se inserta vía vaginal alrededor de la uretra distal, dejándola sin tensión bajo ella de manera que ejerza la suficiente presión sobre la uretra durante los incrementos de la presión abdominal para prevenir el escape de orina ${ }^{45,47}$. Las tasas de curación se sitúan alrededor de $66-91 \%$, con eficacia mantenida más allá de 5 años y una satisfacción del $85 \%$ de las pacientes intervenidas ${ }^{45}$. Los datos disponibles indican también que estas tasas de curación son similares a las obtenidas con procedimientos de colposuspensión abiertos ${ }^{48}$. La complicación intraoperatoria más frecuente es la perforación vesical (en torno a $9 \%$ de los pacientes), aunque también se aprecian disfunciones de vaciado vesical (3-5\% de las pacientes), infecciones urinarias (6-22\%) e hiperactividad vesical "de novo" (3-9\%) $)^{45,48}$. Son raras, no obstante, las erosiones de la malla.

Recientemente se ha introducido una modificación técnica según la cual la malla suburetral es introducida en un plano hori- zontal por debajo de la uretra entre ambos agujeros obturadores de forma percutánea con un tunelizador ${ }^{49}$. Los resultados preliminares son prometedores.

\section{Colposuspensiones}

Las colposuspensiones indicadas en pacientes con IU secundaria a hipermovilidad uretral, se han considerado como el patrón de referencia en el tratamiento quirúrgico de la IUE. Su propósito es estabilizar la uretra estirando los tejidos de la proximidad del cuello vesical y la uretra proximal hacia la cavidad pélvica, detrás de la sínfisis púbica. Se utilizan abordajes tanto abdominales como vaginales $\mathrm{y}$ actualmente laparoscópicos ${ }^{45}$.

La colposuspensión de Burch ha sido la más ampliamente estudiada, con tasas de curación (restablecimiento completo de la continencia) de $73-92 \%$, con unas tasas de éxito (curación o mejoría) de $81-96 \%^{43,48}$. Además estas tasas se mantienen en el tiempo ya que a los 5-10 años el $70 \%$ de las pacientes continúan continentes ${ }^{50}$. Las complicaciones más frecuentes son la disfunción de vaciado vesical (en 2-27\% de las pacientes) y la hiperactividad vesical "de novo" (8-27\%). El grado de satisfacción de las pacientes es alto (82\%).

Una reciente revisión sistemática sobre la efectividad de la colposuspensión laparoscópica ${ }^{51}$ puso de manifiesto que las pacientes sometidas a este procedimiento tenían un $8 \%$ más de riesgo de IU que las tratadas mediante colposuspensión abierta. Las tasas de curación subjetivas (85-100\%) fueron comparables a 6-18 meses de seguimiento, al igual que las complicaciones.

\section{BIBLIOGRAFÍA}

1. Abrams P, Cardozo L, Fall M, Griffiths D, Rosier P, Ulmsten U et al. The standardisation of terminology in lower urinary tract function: report from the standardisation sub-committee of the International Continence Society. Neurourol Urodyn 2002; 21: 167-178.

2. CARdozo L. New developments in the management of stress urinary incontinence. BJU Int 2004; 94 (Supl. 1): 1-3.

3. ReXach Cano L, Verdejo Bravo C. Incontinencia urinaria. Inf Ter Sist Nac Salud 1999; 23: 149-159. 
4. HunskaAr S, Burgio K, Diokno AC, Herzog AR, HJALMAS K, LAPITAN MC. Epidemiology and Natural History of Urinary Incontinence (UI). En: Incontinence 2nd Edition. Abrams P, Cardozo L, Khoury S, Wein A (Eds). Chp 3, pp. 165-202. Plymbridge Distributors Ltd., United Kingdom 2002.

5. Dugan E, Roberts CP, Cohen SJ, Presisser JS, DAVIS CC, BLAND DR et al. Why older community-dwelling adults do not discuss urinary incontinence with their primary care physicians. J Am Geriatr Soc 2001; 49: 462-465.

6. EsPuÑa Pons M. Incontinencia de orina en la mujer. Med Clin (Barc) 2003; 120: 464-472.

7. Hannestad YS, Rortveit G, Sandvik H, HunSKAAR S. A community-based epidemiological survey of female urinary incontinence: the Norwegian EPINCONT study. Epidemiology of Incontinence in the County of Nord-Trondelag. J Clin Epidemiol 2000; 53: 1150-1157.

8. Hunskaar S, Lose G, Sykes D, Voss S. The prevalence of urinary incontinence in women in four European countries. BJU Int 2004; 93: 324-330.

9. NiHIRA MA, HENDERSON N. Epidemiology of urinary incontinence in women. Curr Womens Health Rep 2003; 3: 340-347.

10. LosE G. The burden of stress urinary incontinence. Eur Urol Supplements 2005; 4: 5-10.

11. Grupo Español de Urodinámica y de SINUG. Consenso sobre terminología y conceptos de la función del tracto urinario inferior. Actas Urol Esp 2005; 29: 16-30.

12. Minassian VA, DRUTZ HP, Al-BadR A. Urinary incontinence as a worldwide problem. Int $\mathrm{J}$ Gynaecol Obstet 2003; 82: 327-338.

13. SAndvick H, Seim A, VAnVIK A, HunskaAr S. A severity index for epidemiological surveys of female urinary incontinence: Comparison with 48-hour pad-weighing tests. Neurourol Urodyn 2000; 19: 137-145.

14. Grady D, Brown JS, Vittinghoff E, Applegate W, VARNER E, SNYDER T. Postmenopausal hormones and incontinence: the heart and Estrogen/Progestin Replacement Study. Obstet Gynecol 2001; 97: 116-120.

15. Subak LL, Johnson C, Whitcomb E, Boban D, SAXTON J, BRown JS. Does weight loss improve incontinence in moderately obese women? Int Urogynecol J Pelvic Floor Dysfunct $2002 ; 13: 40-43$.

16. Rortveit G, Hannestad YS, Daltveit AK, HunSKAAR S. Age- and type-dependent effects of parity on urinary incontinence: the Norwegian EPINCONT study. Obstet Gynecol 2001; 98: 1004-1110.
17. RoRtveit G, Daltveit AK, Hannestad YS, HunSKAAR S. Urinary incontinence after vaginal delivery or cesarean section. N Engl J Med 2003; 348: 900-907.

18. RoRtveit G, Hannestad YS, Daltveit AK, HunSKAAR S. Vaginal delivery parameters and urinary incontinence: the Norwegian EPINCONT study. Am J Obstet Gynecol 2003; 189: 1268-1274.

19. Brown JS, Sawaya G, Thom DH, Grady D. Hysterectomy and urinary incontinence: a systematic review. Lancet 2000; 356: 535-539.

20. Contreras Ortiz O. Stress urinary incontinence in the gynecological practice. Int $\mathrm{J}$ Gynecol Obstet 2004; 86 (Suppl. 1): S6-S16.

21. VIKTRUP L, LOSE G. Lower urinary tract symptoms 5 years after the first delivery. Int Urogynecol J 2000; 11: 336-340.

22. Treiber U, Hellerhoff P, Hannig C, May F, HaRTUNG R. Dynamic MRI for evaluation of female pelvis in urinary stress incontinence and pelvic organ prolapse. Eur Urol 2004; 3: 2002.

23. Hannestad YS, LiE RT, Rortveit G, HunskaAR S. Familial risk of urinary incontinence in women: population based cross sectional study. B Med J 2004; 329: 889-891.

24. KELLEHER C. Quality of life and urinary incontinence. En: Textebook of Female Urology and Urogynecology. Cardozo L, Staskin D (Eds). Chp 5: 47-58. Isis Medical Media Ltd, United Kingdom 2001.

25. PAscual MA. Prevalencia y calidad de vida. Prog Obstet Ginecol 2002; 45 (Supl. 1): 2-9.

26. Ertunc D, Tok EC, Pata O, Dilek U, Ozdemir G, DILEK $S$. Is stress urinary incontinence a familial condition? Acta Obstet Gynecol Scand 2004; 83: 912-916.

27. Badia X, Castro D, Conejero J. Validez del cuestionario King's Health para la evaluación de la calidad de vida en pacientes con incontinencia urinaria. Med Clin (Barc) 2000; 114: 654-652.

28. EsPuÑa M, REBollo P. Validación de la versión española del ICIQ-SF. Un cuestionario para evaluar la incontinencia urinaria. Med Clin (Barc) 2004; 122: 288-292.

29. DWyer PL. Differentiating stress urinary incontinence from urge urinary incontinence. Int J Gynaecol Obstet 2004; 86 (Suppl. 1): S17-S24.

30. EsPUÑA Pons M. Incontinencia urinaria de urgencia. Vejiga hiperactiva en la mujer. Prog Obstet Ginecol 2002; 45 (Supl. 1): 41-51.

31. Zudaire-Bergera JJ, Robles-Garcia JE, SAIZSansi A, Rioja-Zuazu J, Regojo-Balboa JM, FerNÁNDEZ-MONTERO JM et al. Incontinencia feme- 
nina: evaluación urodinámica. Rev Med Univ Navarra 2004; 48: 32-36.

32. Pregazzi R, Sartore A, Bortoli P, Grimaldi E, Troiano L, Guaschino S. Perineal ultrasound evaluation of urethral angle and bladder neck mobility in women with stress urinary incontinence. BJOG 2002; 109: 821-827.

33. Lienemann A, Sprenger D, Janssen U, Grosch E, Pellengahr C, ANThuber C. Assessment of pelvic organ descent by use of functional cine-MRI: which reference line should be used? Neurourol Urodyn 2004; 23: 33-37.

34. Hannestad YS, RoRtveit G, Daltveit AK, HunSKAAR S. Are smoking and other lifestyle factors associated with female urinary incontinence? The Norwegian EPINCONT Study. BJOG 2003; 110: 247-254.

35. Bo K. Urinary Incontinence, Pelvic Floor Dysfunction, Exercise and Sport. Sports Medicine 2004; 34: 451-464.

36. Sampselle CM, Palmer MH, Boyington AR, O`Dell KK, Wooldridge L. Prevention of Urinary Incontinence in Adults: PopulationBased Strategies. Nursing Research 2004; 53 (Supl. 6): S61-S67.

37. Wallace SA, Roe B, Williams K, Palmer M. Bladder training for urinary incontinence in adults (Cochrane Review). En: The Cochrane Library, Issue 4, 2004. Chichester, UK: John Wiley \& Sons, Ltd.

38. Hay-Smith EJC, Bø K, Berghmans LCM, HenDRIKS HJM, DE BIE RA, VAN WAALWIJK VAN DOORN ESC. Pelvic floor muscle training for urinary incontinence in women (Cochrane Review). En: The Cochrane Library, Issue 4, 2004. Chichester, UK: John Wiley \& Sons, Ltd.

39. Berghmans lC, Hendriks HJ, De Bie RA, Van WaAlwiJk Van Doorn ES, Bo K, Van KerreBROECK PE. Conservative treatment of urge urinary incontinence in women: a systematic review of randomized clinical trials. BJU Int 2000; 85: 254-263.

40. ANDERSSON KE. Antimuscarinic treatment of overactive bladder. Lancet Neurol 2004; 3; 46-53.
41. OUSLANDER JG. Management of overactive bladder. N Engl J Med 2004; 350: 786-799.

42. Robles García JE, Zudaire Bergera JJ, Rosell Costa D, Berian Polo JM. Nuevos fármacos en el tratamiento de la incontinencia urinaria. Rev Med Univ Navarra 2004; 48: 4349.

43. Kershen RT, Hsieh M. Preview of new drugs for overactive bladder and incontinence: Darifenacin, Solifenacin, Trospium, and Duloxetine. Current Urol Reports 2004; 5: 359-367.

44. Michel MC, OelKe M, Peters SLM. The neurourological connection. Eur Urol Supplements 2005; 4: 18-28.

45. PesCe F. Current management of stress urinary incontinence. BJU Int 2004; 94 (Suppl 1): 8-13.

46. Pickard R, Reaper J, Wyness L, Cody DJ, MCCLINTON S, N'Dow J. Periurethral injection therapy for urinary incontinence in women (Cochrane Review). En: The Cochrane Library, Issue 4, 2004. Chichester, UK: John Wiley \& Sons, Ltd.

47. Chartier-Kastler E. SUI: Management and unmet needs. Eur Urol Supplements 2005; 4: 11-17.

48. Bezerra CA, Bruschini H, Cody DJ. Suburethral sling operations for urinary incontinence in women (Cochrane Review). In: The Cochrane Library, Issue 4, 2004. Chichester, UK: John Wiley \& Sons, Ltd.

49. Delorme E, Droupy S, De Tayrac R, Delmas V. Transobturator tape (Uratape): a new minimally-invasive procedure to treat female urinary incontinence. Eur Urol 2004; 45: 203207.

50. LAPITAN MC, Cody DJ, GRANT AM. Open retropubic colposuspension for urinary incontinence in women (Cochrane Review). En: The Cochrane Library, Issue 4, 2004. Chichester, UK: John Wiley \& Sons, Ltd.

51. Moehrer B, CAREy M, Wilson D. Laparoscopic colposuspension: a systematic review. BJOG 2003; 110: 230-235. 
